\title{
Racial differences in the association of NT-proBNP with risk of incident heart failure in REGARDS
}

Nirav Patel, ${ }^{1}$ Mary Cushman, ${ }^{2}$ Orlando M. Gutiérrez,, ${ }^{3,4}$ George Howard, ${ }^{5}$ Monika M. Safford, ${ }^{6}$ Paul Muntner, ${ }^{4}$ Raegan W. Durant, ${ }^{7}$ Sumanth D. Prabhu, ${ }^{1,8}$ Garima Arora, ${ }^{1}$ Emily B. Levitan, ${ }^{4}$ and Pankaj Arora ${ }^{1,8}$

'Division of Cardiovascular Disease, Department of Medicine, University of Alabama at Birmingham, Birmingham, Alabama, USA. 2Division of Hematology and Oncology, Department of Medicine, Larner College of Medicine at the University of Vermont, Burlington, Vermont, USA. ${ }^{3}$ Division of Nephrology, Department of Medicine, ${ }^{4}$ Department of Epidemiology, and ${ }^{5}$ Department of Biostatistics, University of Alabama at Birmingham, Birmingham, Alabama, USA ${ }^{6}$ Division of General Internal Medicine, Department of Medicine, Weill Cornell Medical College, New York, New York, USA. ${ }^{7}$ Division of Preventive Medicine, Department of Medicine, University of Alabama at Birmingham, Birmingham, Alabama, USA. ${ }^{8}$ Section of Cardiology, Birmingham Veterans Affairs Medical Center, Birmingham, Alabama, USA.

BACKGROUND. Black individuals have lower natriuretic peptide levels and greater risk of heart failure (HF) than White individuals. Higher N-terminal pro-B-type natriuretic peptide (NTproBNP) is associated with increased risk of incident $\mathrm{HF}$, but little information is available in Black individuals. We examined race-specific differences in (a) the association of NT-proBNP with incident $\mathrm{HF}$ and (b) the predictive ability of NT-proBNP for incident HF across BMI and estimated glomerular filtration rate (eGFR) categories.

METHODS. In a prospective case-cohort study, baseline NT-proBNP was measured among 687 participants with incident HF and 2923 (weighted 20,075) non-case randomly selected participants. Multivariable Cox proportional hazard modeling was used to assess the objectives of our study. Clobal Wald $\chi^{2}$ score estimated from multivariable Cox models was used to assess predictive ability of NT-proBNP across BMI and eGFR categories.

RESULTS. In the multivariable model, a doubling of NT-proBNP concentration was associated with greater risk of incident $\mathrm{HF}$ among White individuals (HR: 1.73; 95\% Cl: 1.55-1.94) than Black individuals (HR: 1.51; $95 \% \mathrm{Cl}: 1.34-1.70$ ), with $P_{\text {interaction }}$ by race of 0.024 . Higher NT-proBNP was the strongest predictor of incident HF across all BMI and eGFR categories among White individuals. In contrast, among Black individuals with obesity $\left(B M I \geq 30 \mathrm{~kg} / \mathrm{m}^{2}\right.$ ) or eGFR less than $60 \mathrm{~mL} / \mathrm{min} / 1.73$ $\mathrm{m}^{2}$, the predictive ability of NT-proBNP for incident HF was attenuated.

CONCLUSIONS. The magnitude of the association of higher NT-proBNP with incident HF risk was greater among White individuals than Black individuals. The diminished ability of NT-proBNP to predict the risk of HF in the Black population with obesity or impaired kidney function highlights the need for further investigations.

FUNDING. National Heart, Lung, and Blood Institute; National Institute of Neurological Disorders and Stroke; National Institute on Aging; and the NIH.

honoraria from Amgen and Keryx and grant support from Amgen, Keryx, and ClaxoSmithKline. MMS has received grant support from Amgen.

Copyright: (c) 2019, American Society for Clinical Investigation.

Submitted: May 1, 2019

Accepted: May 24, 2019

Published: June 4, 2019.

\section{Introduction}

Natriuretic peptides (NPs) are hormones produced by the heart (1). Hemodynamic challenges such as pressure overload seen in hypertension and volume overload seen in heart failure (HF) are associated with higher circulating NP levels $(2,3)$. The role of NPs in the diagnosis and prognosis of HF has been well established (4-6). Additionally, population-based studies have concluded that NPs can predict the risk of cardiovascular disease, including incident HF (7-9). However, the information on racial differences in the relationship between N-terminal pro- B-type NP (NT-proBNP) levels and incident HF is small. Study 


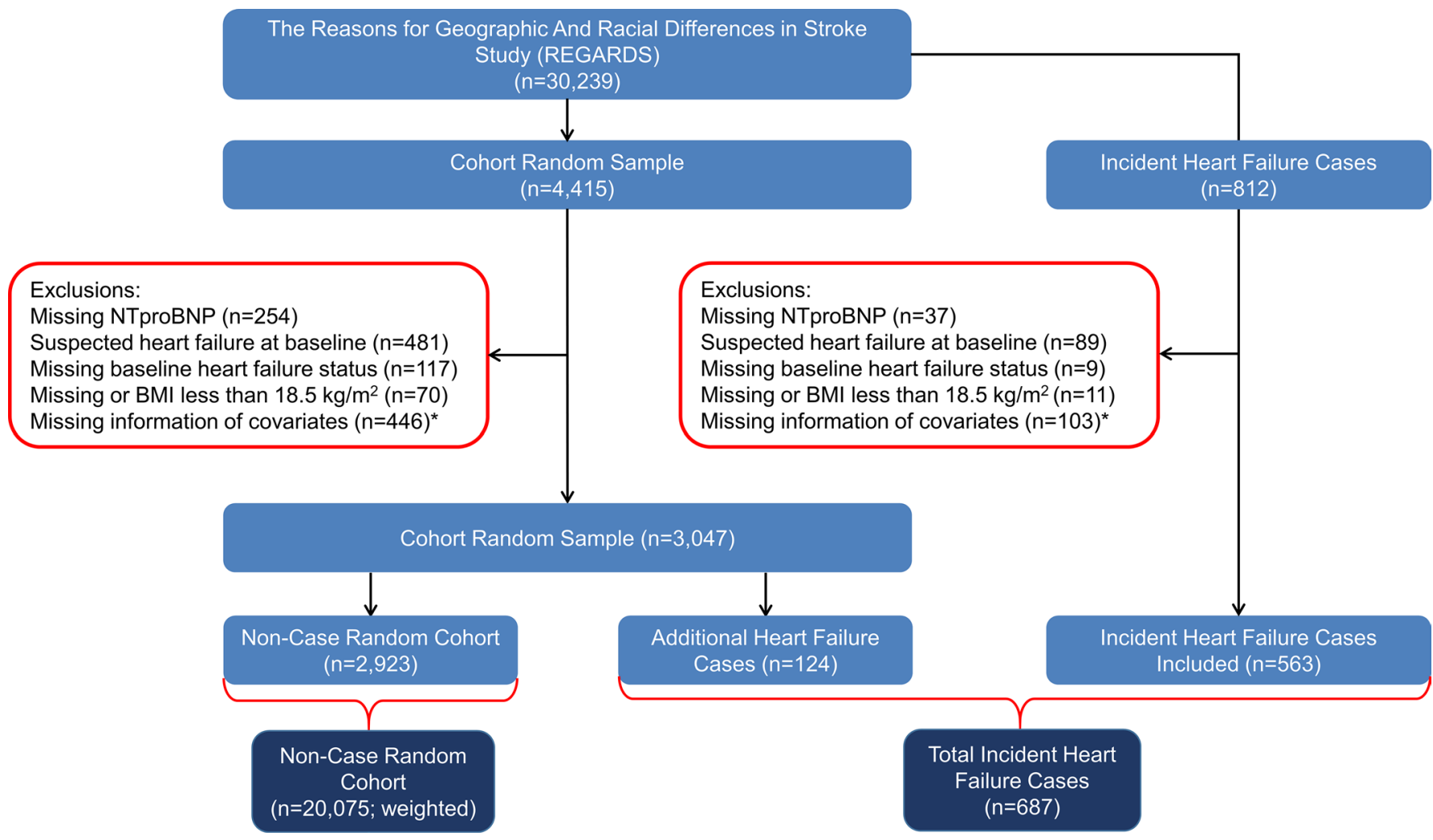

Figure 1. Flow diagram describing the selection of participants for the case-cohort study. *Covariates include age, race, sex, neighborhood socioeconomic status (nSES), tobacco usage, physical activity, alcohol use, systolic blood pressure, use of antihypertensive medications, aspirin use, dyslipidemia, diabetes mellitus, history of stroke or transient ischemic attack (TIA), atrial fibrillation, peripheral vascular disease, history of coronary artery disease, left ventricular hypertrophy, and eGFR.

of racial differences in magnitude of association of NP levels with incident HF is of particular relevance because we and others recently described that Black individuals have significantly lower NP levels as compared with White individuals (10-13).

In addition to race, increasing age, sex, obesity, and impaired renal function affect circulating NP levels (14-18). Prior studies have reported that the association between NP levels and incident HF differed by BMI (19) and estimated glomerular filtration rate (eGFR) categories (20). However, little is known about the relationship of NP levels and incident HF across BMI and eGFR categories among Black individuals, a key racial group at risk for HF. The REasons for Geographic And Racial Differences in Stroke (REGARDS) study offers a unique opportunity to examine race-specific risk associated with NT-proBNP for incident HF, including in subgroups who are obese or have impaired renal function in a large, biracial population of US adults.

We aimed to examine (a) the differences in the relationship of NT-proBNP levels and incident HF by race and (b) whether the risk of incident HF predicted by NT-proBNP levels across BMI and eGFR categories differed by race.

\section{Results}

Study participants. Of the 4415 randomly selected participants, we excluded individuals with missing NT-proBNP $(n=254)$, suspect or missing information on HF status $(n=598)$, and missing BMI or BMI less than $18.5 \mathrm{~kg} / \mathrm{m}^{2}(n=70)$ (i.e., because of fewer participants and possible confounding with other BMI categories). We additionally excluded individuals with missing information about other covariates $(n=$ 446) (Figure 1). There were 124 incident HF cases identified among randomly selected participants. After applying similar exclusion criteria, we excluded 249 individuals from the 812 incident HF cases (Figure 1). These exclusions resulted in including 687 cases of incident HF during follow-up and 2923 participants in the cohort random sample in the study (Figure 1).

Table 1 depicts the baseline characteristics of REGARDS participants in the non-case cohort random sample by race. The proportion of women was higher among Black participants as compared with White 
Table 1. Baseline characteristics in non-case cohort random participants stratified by race from REGARDS

\begin{tabular}{|c|c|c|c|c|}
\hline & & White individuals & Black individuals & $P$ value \\
\hline \multicolumn{2}{|c|}{ Weighted frequency } & 11,872 & 8203 & \\
\hline \multicolumn{2}{|l|}{ Age, years } & $64(58-71)$ & $63(56-69)$ & 0.10 \\
\hline \multirow{3}{*}{ Lifestyle habits } & Current smoker, $n(\%)$ & $1386(11.7)$ & $1373(16.7)$ & $<0.001$ \\
\hline & Current alcohol use, $n(\%)$ & $7271(61.2)$ & $3495(42.6)$ & $<0.001$ \\
\hline & Exercise $>4$ times a week, $n(\%)$ & $4065(34.2)$ & $2261(27.6)$ & $<0.001$ \\
\hline \multirow{2}{*}{ Medication use } & Aspirin, $n(\%)$ & $5625(47.4)$ & $2845(34.7)$ & $<0.001$ \\
\hline & Antihypertensive, $n(\%)$ & $5593(47.1)$ & $5760(70.2)$ & $<0.001$ \\
\hline \multicolumn{2}{|l|}{ Diabetes, $n(\%)$} & $1550(13.1)$ & $2061(25.1)$ & $<0.001$ \\
\hline \multicolumn{2}{|c|}{ Dyslipidemia, $n$ (\%) } & $7153(60.3)$ & $4294(52.3)$ & $<0.001$ \\
\hline \multicolumn{2}{|c|}{ History of stroke or TIA, $n$ (\%) } & $939(7.9)$ & $795(9.7)$ & 0.12 \\
\hline \multicolumn{2}{|l|}{ nSES } & $1.0(-2.2,5.5)$ & $-2.8(-5.1,0.0)$ & $<0.001$ \\
\hline \multicolumn{2}{|c|}{ NT-proBNP, pg/mL } & $71(38-137)$ & $52(25-109)$ & $<0.001$ \\
\hline \multicolumn{2}{|c|}{$\mathrm{eGFR}, \mathrm{mL} / \mathrm{min} / 1.73 \mathrm{~m}^{2}$} & $85(74-95)$ & $94(77-108)$ & $<0.001$ \\
\hline
\end{tabular}

Weighted to original cohort overall. Continuous variables are shown as median and IQR, and categorical variables are shown as $n$ (\%).BMI, body mass index; eGFR, estimated glomerular filtration rate; nSES: neighborhood socioeconomic status at census block level; NT-proBNP, N-terminal pro-B-type natriuretic peptide; PVD, peripheral vascular disease; REGARDS, REasons for Geographic And Racial Differences in Stroke Study; SBP, systolic blood pressure; and TIA, transient ischemic attack.

participants $(61.6 \%$ vs. $49.9 \%$; $P<0.001)$ (Table 1). A higher proportion of Black individuals were current smokers, while a higher proportion of White individuals were current alcohol users (Table 1). Black individuals were more likely to have higher BMI, systolic blood pressure, hypertension, diabetes, and left ventricular hypertrophy as compared with White individuals. In contrast, White individuals were more likely to have dyslipidemia, aspirin use, and coronary artery disease compared with Black individuals (Table 1). The median eGFR was significantly higher among Black participants as compared with White participants (94 [IQR: 77-108] vs. 85 [IQR: 74-95]; $P<0.001$ ) (Table 1). White participants were more likely to live in more economically advantaged neighborhoods compared with Black participants (median $Z$ score [IQR]: $1.0[-2.2,5.5]$ vs. $-2.8[-5.1,0.0], P<0.001$ ) (Table 1). The baseline characteristics of participants in the non-case cohort random sample by tertiles of NT-proBNP as well as participants who developed HF ( $n=$ 687) are presented as Supplemental Tables 1 and 2; supplemental material available online with this article; https://doi.org/10.1172/jci.insight.129979DS1.

NT-proBNP levels and incident HF. The risk of HF rose sharply across tertiles of baseline NT-proBNP (Table 2). Specifically, the multivariable-adjusted HRs for incident HF in the second and third, compared with the first, tertile were 1.77 (95\% CI: 1.29-2.43) and 6.28 (95\% CI: 4.51-8.73), respectively (Table 2). In an unadjusted model, each doubling of NT-proBNP was associated with $86 \%$ greater risk of incident HF (HR: 1.86; 95\% CI: 1.71-2.03) (Table 2). Results were mildly attenuated in the model adjusted for age, sex, and race (HR: 1.69; 95\% CI: 1.56-1.84) and the fully adjusted model (HR: 1.63; 95\% CI: 1.50-1.76). The relationship between NT-proBNP and incident HF was significantly modified by race in the fully adjusted model ( $P_{\text {interaction }}$ $=0.024$ ). Therefore, we further examined the models stratified by race (Table 3). Among 687 incident HF cases, 302 were classified as $\mathrm{HF}$ with reduced ejection fraction ( $\mathrm{HFrEF})$ and 247 as $\mathrm{HF}$ with preserved EF (HFpEF). In the fully adjusted model, we did not observe any effect modification between NT-proBNP and incident $\mathrm{HFrEF}$ or $\mathrm{HFpEF}$ by race $\left(P_{\text {interaction }}=0.10\right)$.

The magnitude of the association between NT-proBNP levels and incident HF was greater in White individuals (HR per doubling of NT-proBNP: 2.09; 95\% CI: 1.85-2.37) than in Black individuals (HR: 1.69; 95\% CI: 1.52-1.89) in the unadjusted model (Table 3). In the multivariable model, the pattern was the same 
Table 2. HR of incident HF by tertiles and per doubling of plasma NT-proBNP

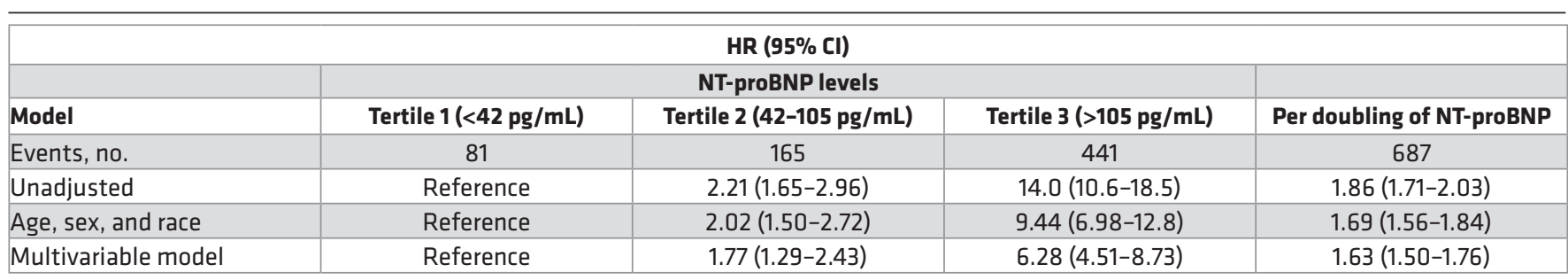

Multivariable Cox regression model including age, sex, race, exercise, smoking, alcohol, BMI, systolic blood pressure, antihypertensive medication, aspirin use, dyslipidemia, diabetes, eGFR, history of stroke, history of TIA, atrial fibrillation, peripheral vascular disease, history of coronary artery disease, left ventricular hypertrophy, and nSES at census block level.

(HR: 1.73; 95\% CI: 1.55-1.94) as in Black individuals (HR: 1.51; 95\% CI: 1.34-1.70) (Table 3). Among White individuals, the multivariable-adjusted HRs for incident $\mathrm{HF}$ in the second and third, compared with first, tertile were 1.51 (95\% CI: 0.95-2.41) and 5.82 (95\% CI: 3.59-9.42), respectively (Table 3). Compared with the first tertile, the multivariable-adjusted HRs for incident $\mathrm{HF}$ in the second and third tertiles were 2.24 (95\% CI: 1.44-3.50) and 6.99 (95\% CI: 4.34-11.3), respectively, among Black individuals (Table 3).

NT-proBNP levels and incident HF by BMI and eGFR categories. Among White participants, the risk of HF per doubling of NT-proBNP was similar across BMI and eGFR categories $\left(P_{\text {interaction }}>0.1\right)$ (Table 4$)$. However, among Black individuals, we observed significant interactions between NT-proBNP and BMI $\left(P_{\text {interaction }}=0.08\right)$ and eGFR $\left(P_{\text {interaction }}<0.001\right)$ categories (Table 4$)$. We further assessed the risk of HF per doubling of NT-proBNP among White and Black individuals with obesity $\left(B M I \geq 30 \mathrm{~kg} / \mathrm{m}^{2}\right)$ or renal dysfunction (eGFR $<60 \mathrm{~mL} / \mathrm{min} / 1.73 \mathrm{~m}^{2}$ ). In multivariable-adjusted models, we observed that the relationship between NT-proBNP and incident HF was significantly modified by race in both obese $\left(P_{\text {interaction }}\right.$ $=0.016)$ and renal dysfunction subgroups $\left(P_{\text {interaction }}=0.007\right)$. The level of significance for the aforementioned interaction terms remained significant $(P<0.1)$ after adjusting for multiple testing using Bonferroni's method. Among individuals with obesity, the magnitude of the association between NT-proBNP and incident HF was greater in White participants (HR: 2.03; 95\% CI: 1.73-2.38) than Black participants (HR: 1.28; 95\% CI: 1.08-1.53). Similarly among individuals with renal dysfunction, the effect size of the relationship between NT-proBNP and incident HF was greater in White participants (HR: 1.78; 95\% CI: 1.46-2.17) as compared with Black participants (HR: 1.35; 95\% CI: 1.17-1.55) (Table 4).

Table 3. Racial differences in the relationship of baseline plasma NT-proBNP with incident HF (by tertiles and per doubling of NT-proBNP levels)

\begin{tabular}{|c|c|c|c|c|}
\hline \multicolumn{5}{|c|}{ HR $(95 \% \mathrm{CI})$} \\
\hline Model & Tertile $1(<42 \mathrm{pg} / \mathrm{mL})$ & Tertile 2 (42-105 pg/mL) & Tertile 3 (>105 pg/mL) & Per doubling of NT-proBNP levels \\
\hline Events, no. & 34 & 88 & 290 & 412 \\
\hline Unadjusted & Reference & $1.40(1.57-3.67)$ & $18.1(12.1-27.1)$ & $2.09(1.85-2.37)$ \\
\hline Age and sex & Reference & $1.74(1.12-2.70)$ & $8.77(5.57-13.8)$ & $1.82(1.63-2.05)$ \\
\hline Events, no. & 47 & 77 & 151 & 275 \\
\hline Unadjusted & Reference & $2.45(1.63-3.70)$ & $12.5(8.38-18.8)$ & $1.69(1.52-1.89)$ \\
\hline Age and sex & Reference & $2.47(1.66-3.70)$ & $10.1(6.69-15.3)$ & $1.61(1.46-1.78)$ \\
\hline Multivariable model & Reference & $2.24(1.44-3.50)$ & $6.99(4.34-11.3)$ & $1.51(1.34-1.70)$ \\
\hline
\end{tabular}

Multivariable Cox regression model including age, sex, exercise, smoking, alcohol, BMI, systolic blood pressure, antihypertensive medication, aspirin use, dyslipidemia, diabetes, eGFR, history of stroke or TIA, atrial fibrillation, peripheral vascular disease history of coronary artery disease, left ventricular hypertrophy, and nSES at census block level. $P=0.024$ for interaction in the association of NT-proBNP with incident HF by race. 
A

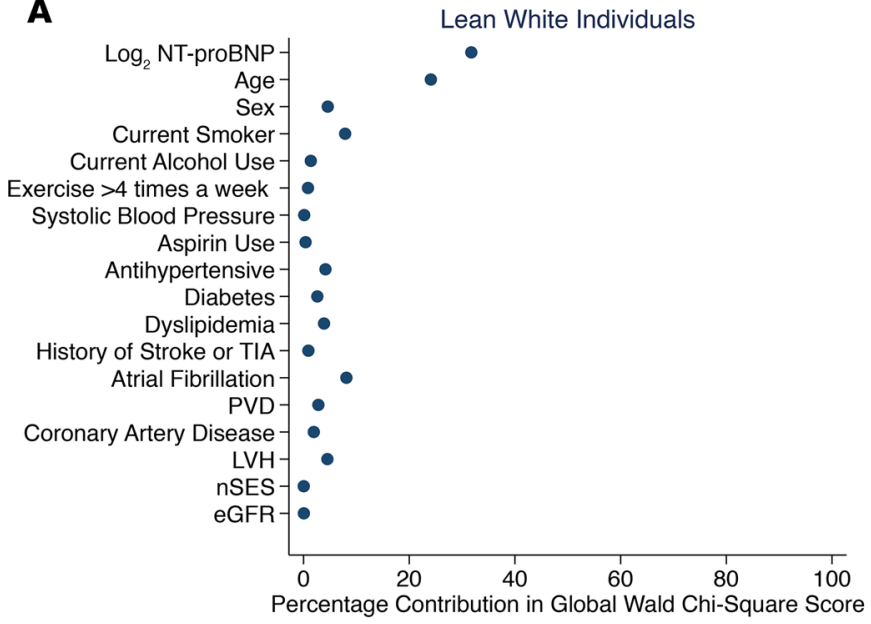

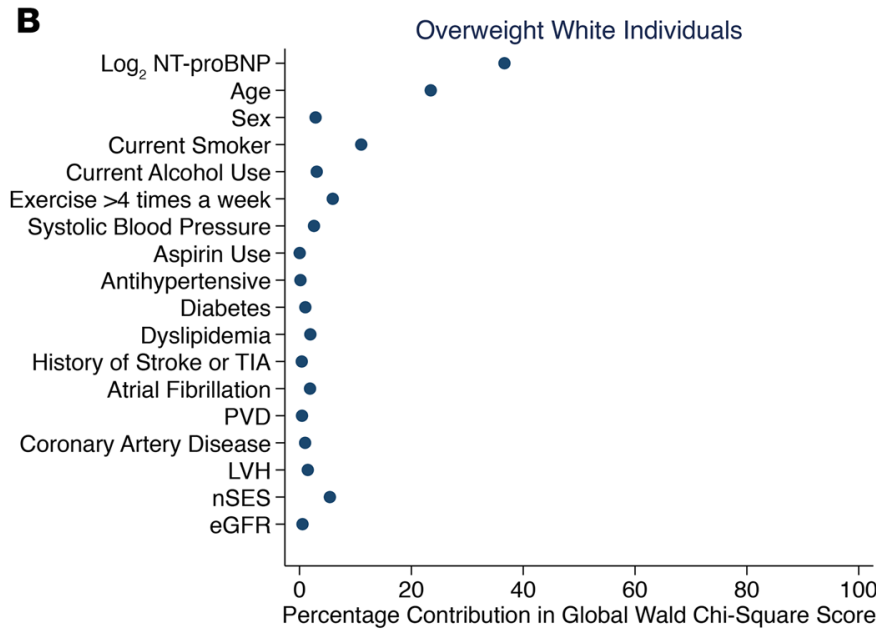

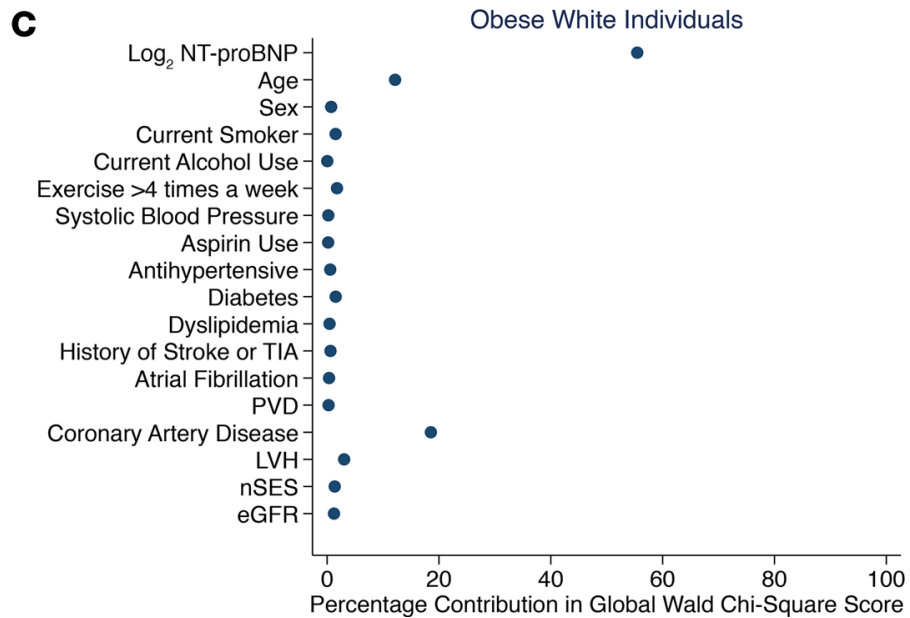

Figure 2. The association between plasma NT-proBNP levels and other factors with incident HF across BMI categories among White individuals. (A) Lean category, BMI $18.5-24.9 \mathrm{~kg} / \mathrm{m}^{2}$. (B) Overweight category, BMI $25-29.9 \mathrm{~kg} / \mathrm{m}^{2}$. (C) Obese category, BMI at least $30 \mathrm{~kg} / \mathrm{m}^{2}$. The panels display the factors ( $y$ axis) and their percentage contribution in the global Wald $\chi^{2}$ score from the multivariable-adjusted Cox model. BMI, body mass index; GFR, glomerular filtration rate; LVH, left ventricular hypertrophy; nSES, neighborhood socioeconomic status at census block level; NT-proBNP, N-terminal pro-B-type natriuretic peptide; PVD, peripheral vascular disease; TIA, transient ischemic attack.

The ranking in the percentage contribution of NT-proBNP to HF risk was examined to assess the predictive ability of NT-proBNP for incident HF across BMI and eGFR categories by race (Figures 2 and 3 across BMI and Figure 4 across eGFR categories by race). In this assessment, a higher rank (i.e., greater percentage contribution in global Wald $\chi^{2}$ score) means the variable is a stronger predictor than variables with lower ranks. In multivariable-adjusted Cox models, among White individuals, higher NT-proBNP was the strongest predictor of incident HF across BMI (Figure 2, A-C) and eGFR categories (Figure 4, A and B) as compared with other covariates. However, the strength of the association between NT-proBNP levels and incident HF was variable across BMI and eGFR categories among Black individuals. Higher NT-proBNP was the strongest predictor of incident HF only among lean and overweight Black individuals (Figure 3, A and B) as well as Black individuals with eGFR at least $60 \mathrm{~mL} / \mathrm{min} / 1.73 \mathrm{~m}^{2}$ (Figure 4C). Among Black individuals with obesity (BMI $\geq 30 \mathrm{~kg} / \mathrm{m}^{2}$ ), current smoking and increasing age were the strongest predictors of incident HF followed by NT-proBNP levels (Figure 3C). Higher NT-proBNP was the second strongest predictor behind dyslipidemia among Black individuals with eGFR less than $60 \mathrm{~mL} / \mathrm{min} / 1.73 \mathrm{~m}^{2}$ (Figure 4D). Furthermore, the incremental value of NT-proBNP levels when added to other covariates was small among Black individuals with obesity (BMI $\geq 30 \mathrm{~kg} / \mathrm{m}^{2}$; C-statistics from 0.75 to 0.76 ) and eGFR less than $60 \mathrm{~mL} / \mathrm{min} / 1.73 \mathrm{~m}^{2}$ (C-statistics from 0.69 to 0.70 ) as assessed by Harrell's C-statistic. 
A

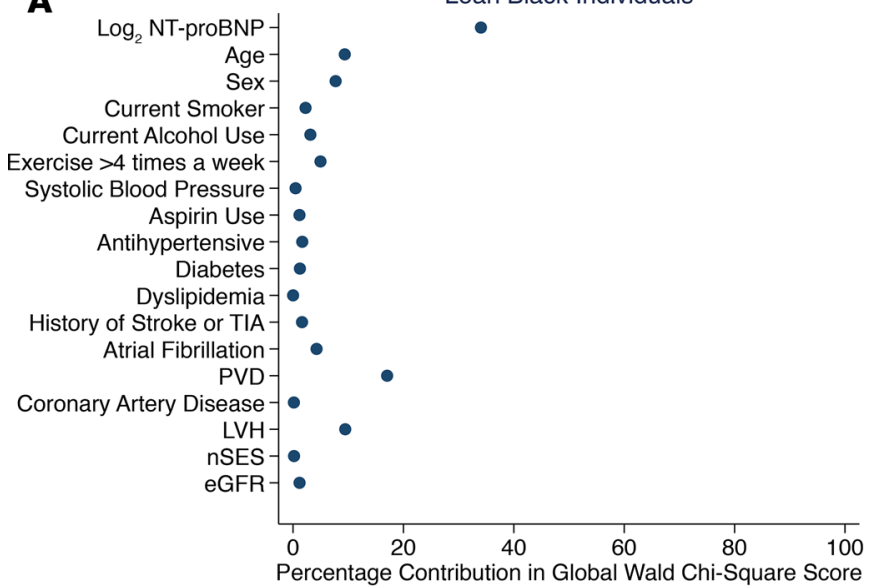

B

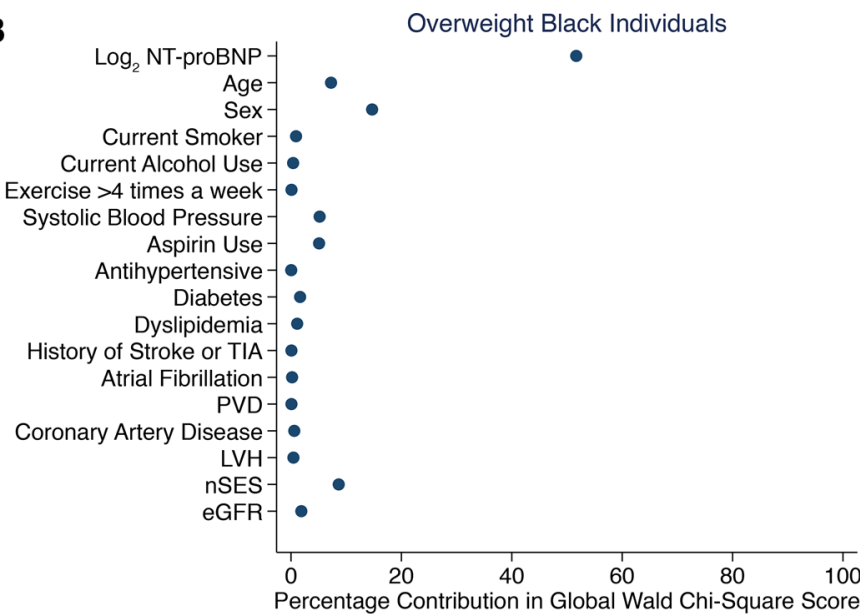

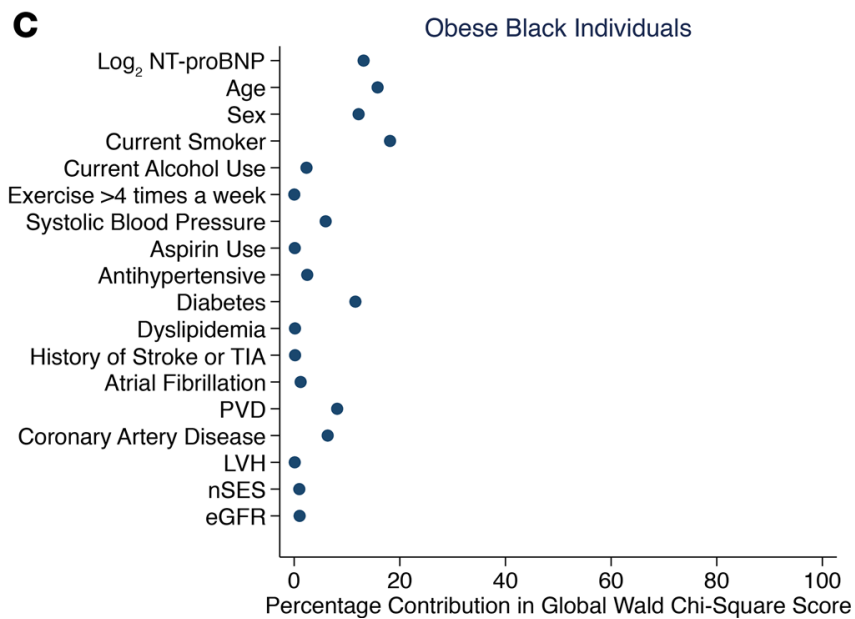

Figure 3. The association between plasma NT-proBNP levels and other factors with incident HF across BMI categories among Black individuals. (A) Lean category, BMI $18.5-24.9 \mathrm{~kg} / \mathrm{m}^{2}$. (B) Overweight category, BMI $25-29.9 \mathrm{~kg} / \mathrm{m}^{2}$. (C) Obese category, BMl at least $30 \mathrm{~kg} / \mathrm{m}^{2}$. The panels display the factors (y axis) and their percentage contribution in the global Wald $\chi^{2}$ score from the multivariable-adjusted Cox model. BMI, body mass index; GFR, glomerular filtration rate; LVH, left ventricular hypertrophy; nSES, neighborhood socioeconomic status at census block level; NT-proBNP, N-terminal pro-B-type natriuretic peptide; PVD, peripheral vascular disease; TIA, transient ischemic attack.

\section{Discussion}

In this population-based study of REGARDS participants, higher NT-proBNP was strongly associated with incident HF independent of sociodemographic and clinical risk factors. The magnitude of the relationship of NT-proBNP levels with incident HF was greater in White individuals than Black individuals (Figure 5). Further, the magnitude of association between NT-proBNP and incident HF was preserved across BMI and eGFR categories among White individuals. However, among Black individuals, an attenuation in the magnitude of the relationship between NT-proBNP and incident HF with increasing BMI and decreasing renal function was observed. The relative importance of NT-proBNP levels was lower among Black individuals with obesity or impaired renal function compared with other covariates (i.e., an attenuated Wald score rank and a marginal 0.01 increment in C-statistics), suggesting that NT-proBNP loses predictive ability for incident HF in the setting of obesity or kidney disease in Black individuals (Figure 5).

The role of NT-proBNP as a biomarker in diagnosis and prognosis of HF has been well established $(4,21)$. Hemodynamic challenges, such as pressure or volume overload, typically stimulate NP secretion, representing the response arm (i.e., a consequence of cardiovascular disease) of the NP system $(2,3)$. The finding that higher NT-proBNP levels were associated with higher risk of incident HF suggests that higher baseline NT-proBNP levels are reflective of subclinical cardiac dysfunction that subsequently manifests itself as overt HF. The relationship of NT-proBNP with incident HF has been previously reported in the Atherosclerosis Risk in Communities (ARIC) (8), Multiethnic Study of Atherosclerosis (MESA) (7), and 
Table 4. Racial differences in the relationship of baseline plasma NT-proBNP with incident HF stratified by BMI and eGFP

\begin{tabular}{|c|c|c|c|c|}
\hline & \multicolumn{2}{|c|}{ White individuals } & \multicolumn{2}{|c|}{ Black individuals } \\
\hline & Events, no. & $\operatorname{HR}(95 \% \mathrm{Cl})^{\mathrm{A}}$ & Events, no. & $\mathrm{HR}(95 \% \mathrm{Cl})^{\mathrm{A}}$ \\
\hline \multicolumn{5}{|c|}{ BMI categories } \\
\hline Overweight $\left(25.0-29.9 \mathrm{~kg} / \mathrm{m}^{2}\right)$ & 178 & $1.81(1.61-2.03)^{\mathrm{B}}$ & 96 & $1.92(1.55-2.38)^{\mathrm{B}}$ \\
\hline Obese $\left(>30.0 \mathrm{~kg} / \mathrm{m}^{2}\right)$ & 126 & $2.03(1.73-2.38)^{\mathrm{B}}$ & 146 & $1.28(1.08-1.53)^{c}$ \\
\hline \multicolumn{5}{|c|}{ eGFR categories } \\
\hline
\end{tabular}

Multivariable Cox regression model including $\log _{2}$ plasma NT-proBNP levels as the independent variable and including age, exercise, smoking, alcohol, BMI (when eGFR category is the exposure variable), systolic blood pressure, antihypertensive medication, aspirin use, dyslipidemia, diabetes, eGFR (when BMI category is the exposure variable), history of stroke, history of TIA, atrial fibrillation, peripheral vascular disease, history of coronary artery disease, left ventricular hypertrophy, and nSES at census block level. $P=0.85$ and $P=0.21$ for interaction in the association of NT-proBNP with incident HF by BMI and eGFR categories, respectively, among White individuals. $P=0.08$ and $P<0.001$ for interaction in the association of NT-proBNP with incident HF by BMI and eGFR categories, respectively, among Black individuals. ${ }^{A} H R$ per doubling $\left(\log _{2}\right)$ of NT-proBNP. ${ }^{B} P<0.001 .{ }^{C} P=0.005$.

other population-based studies $(9,22)$. Our HR estimates for the relationship of NT-proBNP with incident HF were concordant with the previous studies $(7,8)$. We observed that the association of higher NT-proBNP levels with incident HF differed by race, i.e., greater magnitude in HRs among White than Black individuals, albeit the absolute difference in the HRs for incident HF by race was small. These findings may be explained partially by the differences in the NT-proBNP levels between White and Black individuals (10-13). Black individuals have lower NT-proBNP levels than White individuals (10-13). We speculate that Black individuals, when exposed to increased wall stress, such as pressure and volume overload, may not able to mount an appropriate NP response as compared with White individuals. Therefore, the differences in the slope of NP release when exposed to a trigger may explain some of the observed differences in the strength of the relationship of NT-proBNP with incident HF by race. The ARIC and MESA investigators also assessed the association of NT-proBNP with incident HF by race $(7,8)$. The authors of the ARIC study did observe differences in the magnitude of the association of NT-proBNP with incident HF by race (8). However, possibly because of the limited number of Black individuals ( 26\%), ARIC study investigators did not observe a significant interaction by race (8). Similar to the ARIC study (8), investigators from the MESA did not observe any modification of NT-proBNP levels with incident HF by race, again possibly because of the limited number of Black participants ( 24\%) (7). Ours is the first study to our knowledge to report a significant interaction by race in the association of NT-proBNP levels with incident HF. This may be due to the fact that our study is a case-cohort study design and included a large proportion of Black individuals. Additionally, the difference between our findings and those in other cohorts could be due to the changing paradigm of cardiovascular disease risk factors (i.e., decreasing prevalence of ischemic heart disease and increasing prevalence of hypertension and diabetes). This further underscores the significance of assessing the relationship of NT-proBNP with incident HF by race in contemporary cohorts, such as REGARDS. A recent population-based study has suggested that Black race is associated with lower lifetime risk of development of HFpEF but not of HFrEF as compared with other races (23). However, we did not observe any modification in the relationship between NT-proBNP and incident HF subgroups by race, which may be due to limited power to detect this interaction.

Population-based studies have suggested that obese individuals have lower circulating NP levels as compared with lean individuals $(24,25)$, while impaired renal function is associated with higher circulating NP levels $(26,27)$. The association between circulating NP levels and incident HF differed by BMI (19) and renal function categories (20) in other studies. However, neither of the prior studies $(19,20)$ has observed that the association between NP levels and incident HF across BMI and eGFR categories differed by race. Investigators from the ARIC study (19) observed a blunting in the relationship of NT-proBNP levels with incident $\mathrm{HF}$ among individuals with obesity as compared with individuals with normal weight in the overall population. Similar to the ARIC study (19), we observed an attenuation in the association between NT-proBNP levels and incident HF. However, this attenuation was only observed among Black individuals, not in White individuals, in our cohort. The differences between our findings and those of the ARIC study 
A
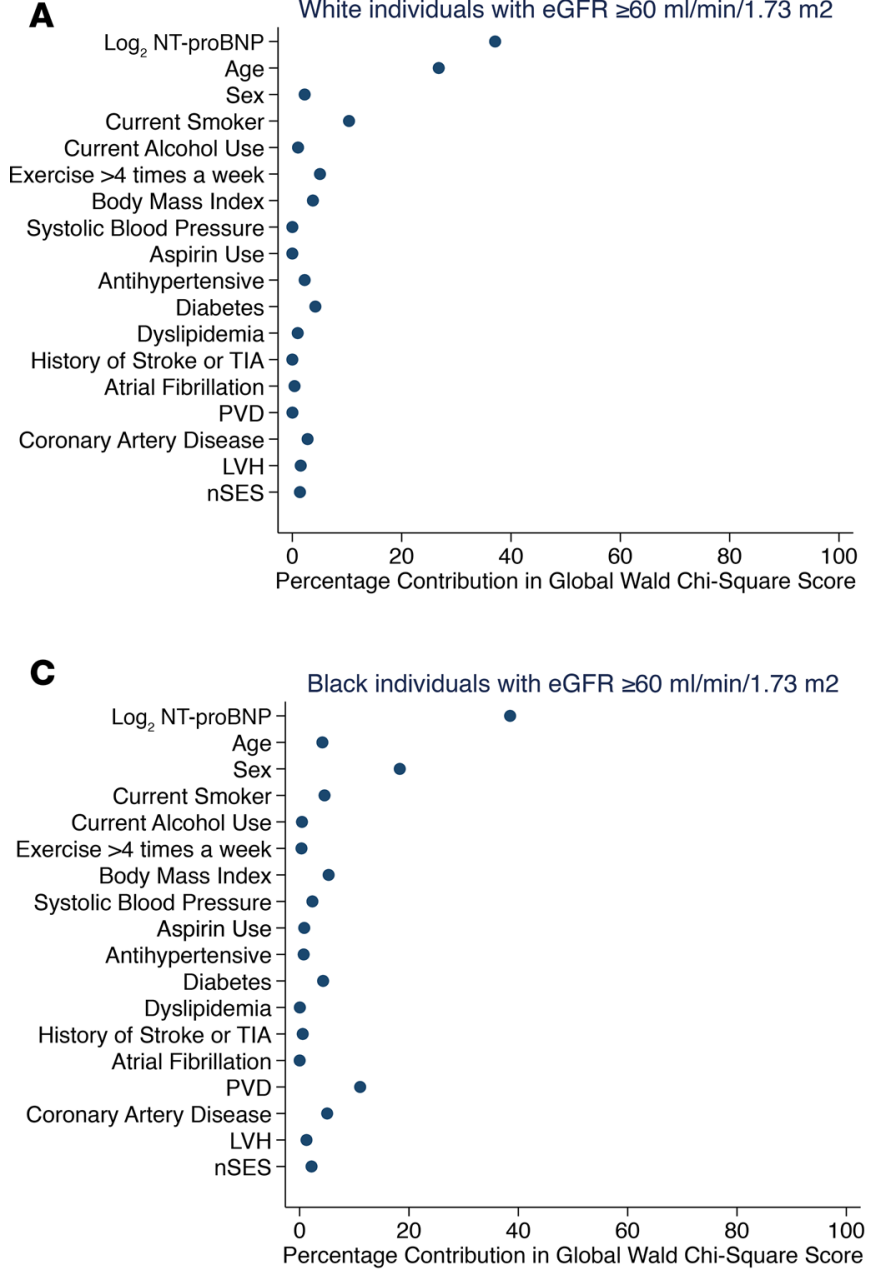

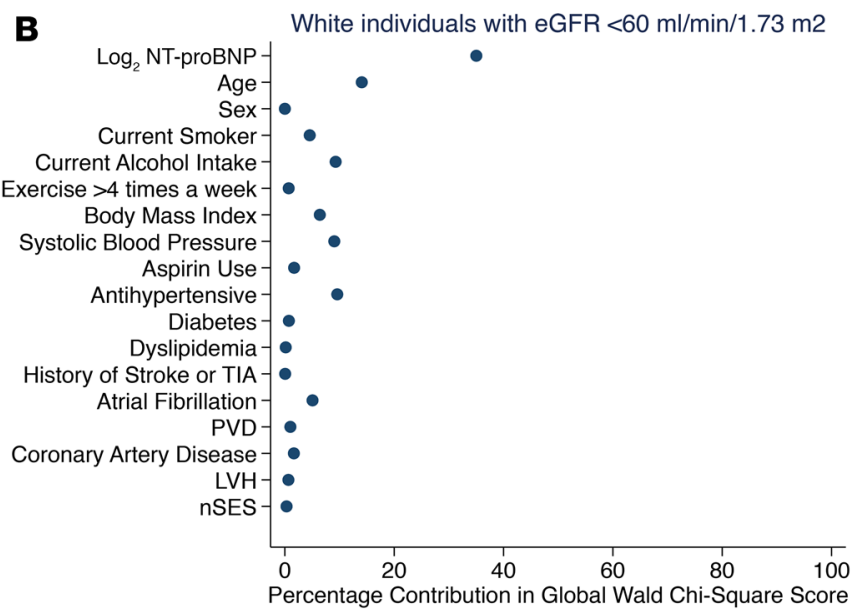

D

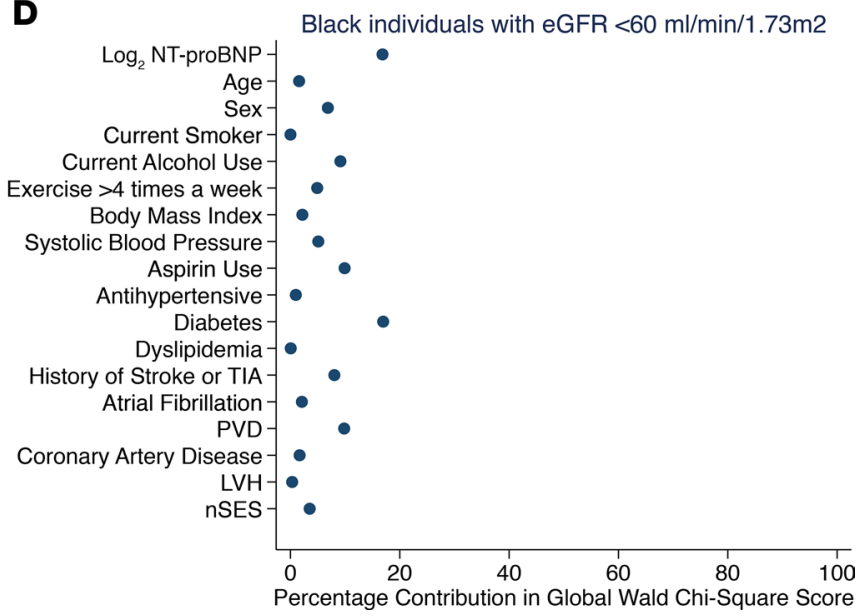

Figure 4. The association between plasma NT-proBNP levels and other factors with incident HF across eGFR categories among White and Black individuals. (A) eGFR at least $60 \mathrm{~mL} / \mathrm{min} / 1.73 \mathrm{~m}^{2}$ and (B) eGFR less than $60 \mathrm{~mL} / \mathrm{min} / 1.73 \mathrm{~m}^{2}$ among White individuals. (C) eGFR at least $60 \mathrm{~mL} / \mathrm{min} / 1.73 \mathrm{~m}{ }^{2}$ and (D) eGFR less than $60 \mathrm{~mL} / \mathrm{min} / 1.73 \mathrm{~m}^{2}$ among Black individuals. The panels display the factors ( $y$ axis) and their percentage contribution in the global Wald $\chi^{2}$ score from the multivariable-adjusted Cox model. eGFR, estimated glomerular filtration rate; LVH, left ventricular hypertrophy; nSES, neighborhood socioeconomic status at census block level; NT-proBNP, N-terminal pro-B-type natriuretic peptide; PVD, peripheral vascular disease; TIA, transient ischemic attack.

(19) may be likely due to the differences in the proportion of Black individuals and characterization of incident HF. The MESA investigators observed a diminished relationship of NT-proBNP levels with incident $\mathrm{HF}$ among individuals with impaired renal function (20). We also observed a weakening in the relationship of the NT-proBNP levels with incident HF albeit among Black individuals.

We observed that NT-proBNP remained a strong predictor of incident HF across BMI and eGFR categories among White individuals but not in Black individuals with obesity and impaired renal function. This observed weaker predictive ability of NT-proBNP with incident HF in these subgroups may be explained by the nonlinear relationship of NT-proBNP with BMI and eGFR among Black individuals (19, 28-30). We recently observed a U-shaped relationship between NT-proBNP levels and BMI among Black individuals (31). Because Black race and obesity both are associated with relatively low NP levels, one can speculate that Black individuals with obesity may be particularly susceptible in their inability to produce an appropriate NP response, leading to its weaker predictive ability for future risk of HF. Similarly, we observed a greater increase in NT-proBNP levels among Black participants with eGFR less than $60 \mathrm{~mL} / \mathrm{min} / 1.73 \mathrm{~m}^{2}$ as compared with White participants (31). The aforementioned finding, as well as a weaker predicative ability of NT-proBNP in Black individuals with impaired renal function, highlights a need for future investigations examining race-specific thresholds for NT-proBNP levels among individuals with impaired renal function.

Public health implications. Our finding that NT-proBNP levels had a weaker association with incident HF in Black individuals likely has important clinical implications. NT-proBNP levels have been used as 
White Individuals

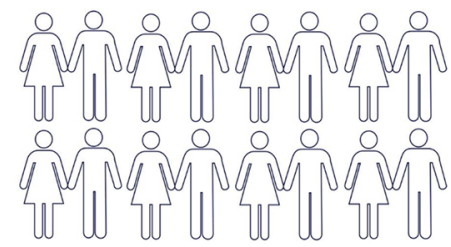

Higher 'Natriuretic Peptides' levels than Black individuals

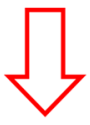

Stronger association of NTproBNP levels with incident heart failure

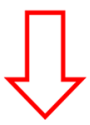

Ability of NT-proBNP for risk prediction of incident heart failure is preserved in white individuals with obesity or renal dysfunction
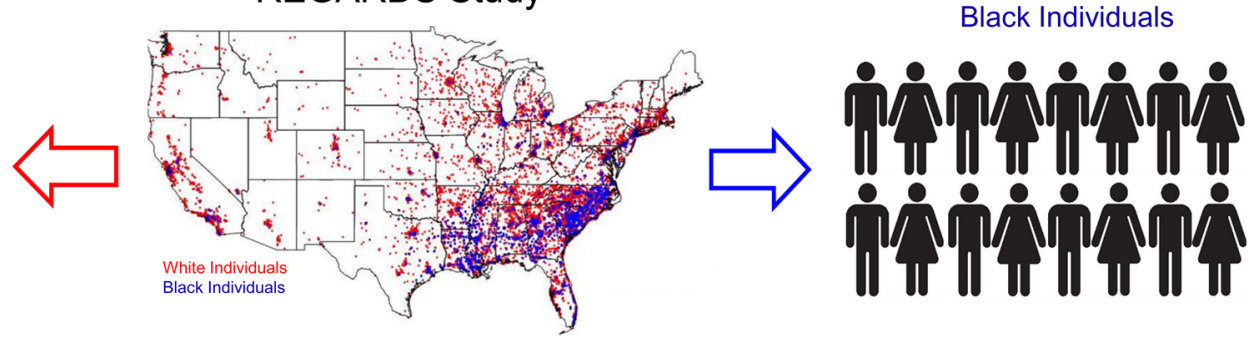

Lower 'Natriuretic Peptides' levels than White individuals

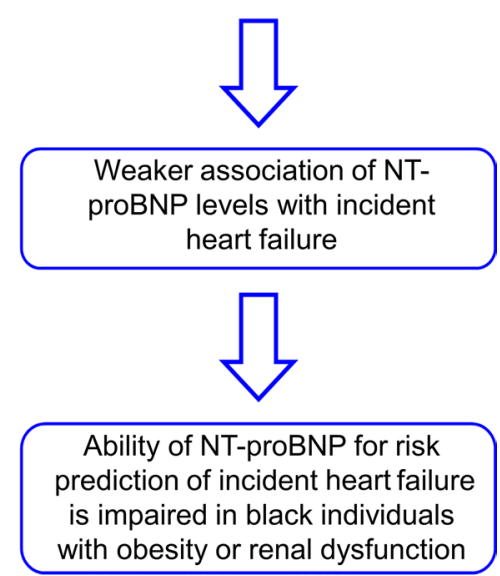

Figure 5. NPs, race, and incident HF. NT-proBNP: N-terminal pro-B-type natriuretic peptide.

an entry/eligibility criterion in multiple recently conducted clinical trials (32-34). Because Black race is associated with relatively low NP levels, this may be a reason behind the marked underrepresentation of Black individuals in the clinical trials where recruitment was based on NP levels (32-34). Our finding of the decrease in the predictive ability of NT-proBNP in Black individuals with severe obesity and impaired renal function may suggest that race-specific cutoff values (i.e., upper limit for normal NP levels) for the diagnosis of HF and when NT-proBNP is used to drive enrollment in clinical trials may be necessary. Currently, the guidelines provide only age-stratified NT-proBNP cutoff values for the diagnosis and prognosis of $\operatorname{HF}(5,35)$. Our findings suggest that to improve the accuracy of risk prediction models using NT-proBNP levels for incident HF, we need to account for other factors that can affect NP levels, such as race, BMI, and eGFR.

Limitations. There are some limitations of our study. Prior studies have shown that NP levels have a genetic basis $(11,13)$. Self-reported characterization of race was used instead of genetic ancestry information markers in this study. The results of the current study cannot be generalized to other racial or ethnic groups because the REGARDS study included only non-Hispanic White and Black individuals. We used medication-based criteria to identify the presence of suspected HF at baseline instead of clinical diagnosis, which likely overestimated the prevalence of baseline HF. Residual confounding because of unmeasured confounders is always possible, in spite of our inclusion of many of the factors that are known to affect NP levels. Finally, we used baseline NT-proBNP levels rather than serial measurements, which precludes study of race-specific trajectories of NT-proBNP in relation to incident $\mathrm{HF}$.

Conclusions. In conclusion, we showed that NT-proBNP levels are associated with incident HF in a large, biracial, population-based study. The relationship of NT-proBNP levels with incident HF differed by race, showing a greater magnitude among White than Black individuals. The risk of HF predicted by NT-proBNP levels was preserved across BMI and eGFR categories among White individuals. However, the predictive ability of NT-proBNP for incident HF was diminished with obesity and impaired renal function among Black individuals. Besides age, important factors such as race, BMI, and eGFR should be considered while assessing and interpreting risk prediction models using NT-proBNP levels for incident HF. 


\section{Methods}

Study population. The REGARDS study was designed to investigate racial and geographic differences in stroke mortality (36). It recruited non-Hispanic White individuals and Black individuals 45 years of age or older from the contiguous United States from 2003 to 2007 (36). Detailed information on the study design, including inclusion exclusion criteria and the recruitment process, has been published (36). A computer-assisted telephone interview followed by an in-home visit were performed to collect information on demographic, socioeconomic, and anthropometric factors; medical history; and medications (36). Blood and urine were also sampled during the in-home visit and stored in a central laboratory (36).

The primary exposure variable in this study was plasma NT-proBNP level. As described previously, NT-proBNP measurement was performed using an automated electrochemiluminescence immunoassay (Roche Elecsys 2010) in baseline plasma samples (10). The intra- and interassay coefficients of variation were less than $2 \%$ and $5 \%$, respectively.

Outcome of interest. The outcome of interest was incident HF hospitalization. Study participants were followed up every 6 months via telephone to detect hospitalizations (37). Each suspected heart disease hospitalization was adjudicated by 2 clinicians by assessing medical records $(37,38)$. Any disagreement between 2 adjudicators was resolved by discussion among the team members. Adjudication of a HF hospitalization was based on clinical signs and symptoms, laboratory studies (including B-type NP or NT-proBNP), and assessment of left ventricular function. An assessment of left ventricular function was based on echocardiography, which was performed during the hospitalization and reported in the medical records. Based on the results of echocardiography, HF was subclassified into (a) HFrEF with left ventricular EF less than 40\%, (b) HFpEF with left ventricular EF greater than 50\%, (c) HF with midrange EF (40\%-50\%), and (d) undetermined category. HF assessment included screening for the following signs and symptoms: abnormal jugular vein distension, pulmonary rales, cardiomegaly, central venous pressure greater than $16 \mathrm{mmHg}$, hepatomegaly, pleural effusion, heart rate greater than $120 \mathrm{bpm}$, and greater than or equal to $4.5-\mathrm{kg}$ weight loss in 5 days with diuresis, paroxysmal nocturnal dyspnea, orthopnea, nocturnal cough, and exertional dyspnea.

Case-cohort study. A case-cohort study design was used $(39,40)$. This approach provides an unbiased estimate of the relative hazard of an outcome without requiring measurement of biomarkers in all participants and without compromising the power of large cohort studies (40). Cases included all participants who developed an incident HF during follow-up through December 31, 2015. After excluding participants with suspected HF at baseline, participants who experienced an adjudicated hospitalization for HF during follow-up from baseline through December 31, 2015, were included as cases (Figure 1). Suspected HF was determined by the use of HF-related medications at the baseline visit, i.e., taking digoxin in the absence of atrial fibrillation, carvedilol, spironolactone, hydralazine with isosorbide mononitrate/dinitrate, or loop diuretics or taking angiotensin-converting enzyme inhibitors or an angiotensin II receptor blocker with a beta blocker in the absence of hypertension. This approach had more than $99 \%$ negative predictive value for excluding prior HF hospitalization compared to Medicare claims.

The cohort random sample (comparison group) was selected using stratified sampling to ensure sufficient representation of high-risk groups (41). All participants with at least 1 follow-up contact $(n=29,653)$ were categorized into 20 strata based on age (45-54, 55-64, 65-74, 75-84, or 85 or more years), race (Black or White), and sex (male or female). In each stratum, participants were randomly selected to fulfill the desired distribution: 50\% Black, 50\% White; 50\% female, 50\% male; and 20\% ages $45-54,20 \%$ ages $55-64$, $25 \%$ ages $65-74,25 \%$ ages $75-84$, and $10 \%$ ages 85 or higher. For this analysis we excluded individuals with prevalent $\mathrm{HF}$ in the cohort random sample.

Covariates of interest. Baseline characteristics, such as age, sex, race, smoking status, alcohol use, and exercise, were self-reported. BMI was measured during the in-home visit as weight in kilograms divided by height in meters squared. We divided the study participants into lean (i.e., BMI $\geq 18.5<25 \mathrm{~kg} / \mathrm{m}^{2}$ ), overweight (i.e., BMI $\geq 25<30 \mathrm{~kg} / \mathrm{m}^{2}$ ), and obese (i.e., BMI $\geq 30 \mathrm{~kg} / \mathrm{m}^{2}$ ) categories. The average of 2 blood pressure measurements taken 5 minutes apart was used to assess systolic blood pressure. Hypertension was considered present if systolic blood pressure was at least $140 \mathrm{mmHg}$, diastolic blood pressure was at least $90 \mathrm{mmHg}$, or the participant reported current use of antihypertensive medication. Aspirin use was self-reported. Diabetes was defined as self-reported current use of oral diabetes medications or of insulin or fasting glucose at least $126 \mathrm{mg} /$ $\mathrm{dL}$ (or nonfasting glucose $\geq 200 \mathrm{mg} / \mathrm{dL}$ among those who did not fast). Dyslipidemia was defined as total cholesterol at least $240 \mathrm{mg} / \mathrm{dL}$, LDL-cholesterol at least $160 \mathrm{mg} / \mathrm{dL}$, HDL-cholesterol no more than $40 \mathrm{mg} / \mathrm{dL}$, 
or self-reported medication use for cholesterol levels. History of stroke or transient ischemic attack (TIA) was self-reported. Atrial fibrillation was defined by self-report or from the presence of atrial fibrillation on the electrocardiogram obtained at the in-home visit. History of coronary artery disease was considered present if the participant reported a past myocardial infarction, coronary bypass grafting, angioplasty, or coronary stenting or had evidence of myocardial infarction on the electrocardiogram at baseline. A history of peripheral vascular disease was considered present if the participant reported a leg amputation or any surgery to fix the arteries in the legs. The presence of left ventricular hypertrophy was determined using Sokolow-Lyon voltage criteria $(42,43)$. Geocoded addresses were used to calculate nSES among REGARDS participants using US census data (44). The nSES score was calculated based on 6 variables as described previously $(44,45)$. The nSES score was summarized based on a $Z$ score (ranging from -11.8 to 29.0 , with higher scores meaning higher nSES, i.e., most advantaged neighborhood) using the aforementioned variables (45). The Chronic Kidney Disease Epidemiology Collaboration equation was used to generate eGFR (46) and was categorized as eGFR of at least 60 $\mathrm{mL} / \mathrm{min} / 1.73 \mathrm{~m}^{2}$ or less than $60 \mathrm{~mL} / \mathrm{min} / 1.73 \mathrm{~m}^{2}$.

Statistics. Statistical analyses were conducted using SAS 9.4 (SAS Institute Inc.). Within the cohort random sample, baseline characteristics were calculated using weights to account for the stratified sampling design by tertiles of NT-proBNP.

Cox proportional hazards models for case-cohort studies were used to estimate the association of NT-proBNP (modeled as a continuous variable and in tertiles) with incident HF (40). For the continuous models, we used $\log _{2}$-transformed NT-proBNP to calculate the HR for incident HF per doubling of NT-proBNP level. In the analyses with NT-proBNP modeled in tertiles, participants with the lowest tertile were chosen as a reference group. We constructed unadjusted models, followed by age, race, and sex-adjusted models and then multivariable-adjusted models to assess the relationship of NT-proBNP with incident HF. The covariates included in the multivariable models were selected based on previous studies demonstrating their association with NT-proBNP levels: (10-13, 47-49) age, race, sex, nSES, tobacco use, physical activity, alcohol use, BMI, systolic blood pressure, use of antihypertensive medications, aspirin use, dyslipidemia, diabetes mellitus, history of stroke or TIA, atrial fibrillation, peripheral vascular disease, history of coronary artery disease, left ventricular hypertrophy, and eGFR. In the multivariable models using NT-proBNP levels as a continuous variable, we further examined for effect modification by race by using a multiplicative interaction term (i.e., race $\times \log _{2}$ NT-proBNP).

The relationship between $\log _{2}$ NT-proBNP and incident HF across BMI and eGFR categories by race was also examined using multivariable Cox proportional hazard models. The following covariates were included in the multivariable models: age, sex, nSES, lifestyle habits (tobacco usage and physical activity), alcohol use, BMI (when eGFR was the exposure variable), systolic blood pressure, use of antihypertensive medications, aspirin use, dyslipidemia, diabetes mellitus, history of stroke or TIA, atrial fibrillation, peripheral vascular disease, history of coronary artery disease, left ventricular hypertrophy, and eGFR (when BMI was the exposure variable). Multiplicative interaction terms (i.e., BMI $\times \log _{2}$ NT-proBNP and eGFR $\times \log _{2}$ NT-proBNP) were used to assess any modification in the association of NT-proBNP with incident HF by BMI and levels of kidney function within each racial group.

The predictive ability of NT-proBNP and other covariates for incident HF in each BMI and eGFR category by race was further assessed using Wald's test statistics (50). The relative importance of NT-proBNP and other covariates for predicting incident HF across BMI and eGFR categories with corrected degrees of freedom allocated to each covariate was depicted by ranking the percentage contribution of the variable-specific Wald's test out of the global Wald $\chi^{2}$ score (i.e., Wald statistics) (51). Ranking of the percentage contribution of $\log _{2}$ NT-proBNP and other variables in the final model was calculated using the following formula: (the $\chi^{2}$ score of the predictor variable/the global $\chi^{2}$ score) $\times 100$. If an attenuation in the ranking of the percentage contribution of NT-proBNP compared with other variables was detected within any of the BMI and eGFR categories, the incremental value of NT-proBNP levels for incident HF was evaluated with Harrell's C-statistic for that model $(52,53)$. First, we ran a Cox regression model with all the covariates except $\log _{2}$ NT-proBNP and noted the C-statistic. Then we added $\log _{2}$ NT-proBNP and reran the Cox models. The difference in C-statistics between these 2 models was calculated. The level of statistical significance was set at $P<0.05$, and all $P$ values were 2 tailed. $P<0.1$ was considered significant for interactions.

Study approval. All participants provided written informed consent, and the institutional review boards from each participating center approved the REGARDS study protocol. A full list of institutions can be found at http://www.regardsstudy.org. 


\section{Author contributions}

NP and PA had full access to all of the data in the study and take responsibility for the integrity of the data and the accuracy of the data analysis. NP, GA, and PA conceived and designed the study. NP, MC, OMG, EBL, GA, and PA acquired, analyzed, and interpreted data. NP, MC, OMG, GA, and PA drafted the manuscript. NP, MC, OMG, GH, MMS, PM, RWD, SDP, EBL, GA, and PA provided critical revision of the manuscript for important intellectual content. NP and PA performed statistical analysis. PA obtained funding. MC, OMG, GH, MMS, PM, RWD, SDP, EBL, GA, and PA provided administrative, technical, and material support. MC, OMG, GH, MMS, PM, RWD, SDP, EBL, GA, and PA supervised the study.

\section{Acknowledgments}

We thank investigators, staff, and participants of the REGARDS study for their valuable contributions. A full list of investigators and institutions can be found at http://www.regardsstudy.org. This research project is supported by cooperative agreement U01-NS041588 co-funded by the National Institute of Neurological Disorders and Stroke (NINDS) and the National Institute on Aging (NIA), NIH, Department of Health and Human Services. The content is solely the responsibility of the authors and does not necessarily represent the official views of the NINDS or the NIA. Representatives of the NINDS were involved in the review of the manuscript but were not directly involved in the collection, management, analysis or interpretation of the data. Additional support was provided by grant R01-HL080477 from the National Heart, Lung, and Blood Institute. Nirav Patel is supported by NIH grant 5T32HL129948-02. Pankaj Arora is supported by NIH Mentored Patient-Oriented Research Award 1K23HL146887-01.

Address correspondence to: Pankaj Arora, 1670 University Blvd., Volker Hall B140, University of Alabama at Birmingham, Birmingham, Alabama 35294-0019, USA. Phone: 205.996.6630; Email: parora@uabmc.edu.

1. Levin ER, Gardner DG, Samson WK. Natriuretic peptides. N Engl J Med. 1998;339(5):321-328.

2. Mukoyama M, et al. Increased human brain natriuretic peptide in congestive heart failure. N Engl J Med. 1990;323(11):757-758.

3. Gottlieb SS, Kukin ML, Ahern D, Packer M. Prognostic importance of atrial natriuretic peptide in patients with chronic heart failure. J Am Coll Cardiol. 1989;13(7):1534-1539.

4. Maisel AS, et al. Rapid measurement of B-type natriuretic peptide in the emergency diagnosis of heart failure. $N$ Engl J Med. 2002;347(3):161-167.

5. Januzzi JL, et al. The N-terminal Pro-BNP investigation of dyspnea in the emergency department (PRIDE) study. Am J Cardiol. 2005;95(8):948-954.

6. Rothenburger M, et al. Aminoterminal pro type B natriuretic peptide as a predictive and prognostic marker in patients with chronic heart failure. J Heart Lung Transplant. 2004;23(10):1189-1197.

7. Choi EY, et al. N-terminal pro-B-type natriuretic peptide, left ventricular mass, and incident heart failure: Multi-Ethnic Study of Atherosclerosis. Circ Heart Fail. 2012;5(6):727-734.

8. Agarwal SK, et al. Prediction of incident heart failure in general practice: the Atherosclerosis Risk in Communities (ARIC) Study. Circ Heart Fail. 2012;5(4):422-429.

9. Wang TJ, et al. Plasma natriuretic peptide levels and the risk of cardiovascular events and death. N Engl J Med. 2004;350(7):655-663.

10. Bajaj NS, et al. Racial differences in plasma levels of N-terminal pro-B-type natriuretic peptide and outcomes: the Reasons for Geographic and Racial Differences in Stroke (REGARDS) study. JAMA Cardiol. 2018;3(1):11-17.

11. Gupta DK, et al. Differences in natriuretic peptide levels by race/ethnicity (from the multi-ethnic study of atherosclerosis). Am $J$ Cardiol. 2017;120(6):1008-1015.

12. Gupta DK, de Lemos JA, Ayers CR, Berry JD, Wang TJ. Racial differences in natriuretic peptide levels: the Dallas Heart Study. JACC Heart Fail. 2015;3(7):513-519.

13. Gupta DK, et al. Racial differences in circulating natriuretic peptide levels: the atherosclerosis risk in communities study. $J A m$ Heart Assoc. 2015;4(5):e001831.

14. Arora P, et al. Weight loss, saline loading, and the natriuretic peptide system. J Am Heart Assoc. 2015;4(1):e001265.

15. Azevedo A, Bettencourt P, Barros H. Demographic, clinical and echocardiographic determinants of B-type natriuretic peptide plasma concentration. A population-based study. Rev Port Cardiol. 2007;26(2):105-113.

16. Raymond I, et al. The influence of age, sex and other variables on the plasma level of N-terminal pro brain natriuretic peptide in a large sample of the general population. Heart. 2003;89(7):745-751.

17. Anwaruddin S, et al. Renal function, congestive heart failure, and amino-terminal pro-brain natriuretic peptide measurement: results from the ProBNP Investigation of Dyspnea in the Emergency Department (PRIDE) Study. J Am Coll Cardiol. 2006;47(1):91-97.

18. Manzano-Fernández S, et al. Impact of kidney dysfunction on plasma and urinary N-terminal pro-B-type natriuretic peptide in patients with acute heart failure. Congest Heart Fail. 2010;16(5):214-220.

19. Ndumele CE, et al. N-terminal pro-brain natriuretic peptide and heart failure risk among individuals with and without obesity: The Atherosclerosis Risk in Communities (ARIC) Study. Circulation. 2016;133(7):631-638.

20. Syed D, et al. Association between $\mathrm{N}$-terminal pro-brain natriuretic peptide levels, glomerular filtration rate, and heart failure in 
the Multi-Ethnic Study of Atherosclerosis. J Integr Cardiol. 2018;4(3):1-7.

21. Maisel AS, et al. Impact of age, race, and sex on the ability of B-type natriuretic peptide to aid in the emergency diagnosis of heart failure: results from the Breathing Not Properly (BNP) multinational study. Am Heart J. 2004;147(6):1078-1084.

22. Kistorp C, Raymond I, Pedersen F, Gustafsson F, Faber J, Hildebrandt P. N-terminal pro-brain natriuretic peptide, C-reactive protein, and urinary albumin levels as predictors of mortality and cardiovascular events in older adults. JAMA. 2005;293(13):1609-1616.

23. Pandey A, et al. Sex and race differences in lifetime risk of heart failure with preserved ejection fraction and heart failure with reduced ejection fraction. Circulation. 2018;137(17):1814-1823.

24. Wang TJ, et al. Impact of obesity on plasma natriuretic peptide levels. Circulation. 2004;109(5):594-600.

25. Horwich TB, Hamilton MA, Fonarow GC. B-type natriuretic peptide levels in obese patients with advanced heart failure. $J$ Am Coll Cardiol. 2006;47(1):85-90.

26. Takase H, Dohi Y. Kidney function crucially affects B-type natriuretic peptide (BNP), N-terminal proBNP and their relationship. Eur J Clin Invest. 2014;44(3):303-308.

27. Wang AY, Lai KN. Use of cardiac biomarkers in end-stage renal disease. J Am Soc Nephrol. 2008;19(9):1643-1652.

28. Fox ER, et al. Relation of obesity to circulating B-type natriuretic peptide concentrations in Blacks: the Jackson Heart Study. Circulation. 2011;124(9):1021-1027.

29. Tsutamoto T, et al. Relationship between renal function and plasma brain natriuretic peptide in patients with heart failure. $J A m$ Coll Cardiol. 2006;47(3):582-586.

30. Das SR, et al. Association between renal function and circulating levels of natriuretic peptides (from the Dallas Heart Study). Am J Cardiol. 2008;102(10):1394-1398.

31. Patel N, et al. Race-based demographic, anthropometric and clinical correlates of N-terminal-pro B-type natriuretic peptide. Int J Cardiol. 2019;286:145-151

32. McMurray JJ, et al. Angiotensin-neprilysin inhibition versus enalapril in heart failure. N Engl J Med. 2014;371(11):993-1004

33. Felker GM, et al. Effect of natriuretic peptide-guided therapy on hospitalization or cardiovascular mortality in high-risk patients with heart failure and reduced ejection fraction: A Randomized Clinical Trial. JAMA. 2017;318(8):713-720.

34. Felker GM, HasselBlad V, Hernandez AF, O'Connor CM. Biomarker-guided therapy in chronic heart failure: a meta-analysis of randomized controlled trials. Am Heart J. 2009;158(3):422-430.

35. Yancy CW, et al. 2013 ACCF/AHA guideline for the management of heart failure: a report of the American College of Cardiology Foundation/American Heart Association Task Force on Practice Guidelines. J Am Coll Cardiol. 2013;62(16):e147-e239.

36. Howard VJ, et al. The reasons for geographic and racial differences in stroke study: objectives and design. Neuroepidemiology. 2005;25(3):135-143.

37. Levitan EB, et al. Medical therapy following hospitalization for heart failure with reduced ejection fraction and association with discharge to long-term care: a cross-sectional analysis of the REasons for Geographic And Racial Differences in Stroke (REGARDS) population. BMC Cardiovasc Disord. 2017;17(1):249.

38. Sterling MR, et al. The prevalence of cognitive impairment among adults with incident heart failure: the "Reasons for Geographic and Racial Differences in Stroke" (REGARDS) study. J Card Fail. 2019;25(2):130-136.

39. Prentice RL. A case-cohort design for epidemiologic cohort studies and disease prevention trials. Biometrika. 1986;73(1):1-11.

40. Barlow WE, Ichikawa L, Rosner D, Izumi S. Analysis of case-cohort designs. J Clin Epidemiol. 1999;52(12):1165-1172.

41. Cushman M, et al. N-terminal pro-B-type natriuretic peptide and stroke risk: the reasons for geographic and racial differences in stroke cohort. Stroke. 2014;45(6):1646-1650.

42. Antikainen R, et al. The determinants of left ventricular hypertrophy defined by Sokolow-Lyon criteria in untreated hypertensive patients. J Hum Hypertens. 2003;17(3):159-164.

43. Sokolow M, Lyon TP. The ventricular complex in left ventricular hypertrophy as obtained by unipolar precordial and limb leads Am Heart J. 1949;37(2):161-186.

44. Diez Roux AV, et al. Neighborhood of residence and incidence of coronary heart disease. NEngl J Med. 2001;345(2):99-106.

45. Howard VJ, et al. Neighborhood socioeconomic index and stroke incidence in a national cohort of Blacks and Whites. Neurology. 2016;87(22):2340-2347.

46. Levey AS, et al. A new equation to estimate glomerular filtration rate. Ann Intern Med. 2009;150(9):604-612.

47. Vart P, et al. SES, heart failure, and N-terminal pro-B-type natriuretic peptide: the Atherosclerosis Risk in Communities study. Am J Prev Med. 2018;54(2):229-236.

48. Jug B, Sebestjen M, Sabovic M, Keber I. Clopidogrel is associated with a lesser increase in NT-proBNP when compared to aspirin in patients with ischemic heart failure. J Card Fail. 2006;12(6):446-451.

49. Wang TJ, Larson MG, Keyes MJ, Levy D, Benjamin EJ, Vasan RS. Association of plasma natriuretic peptide levels with metabolic risk factors in ambulatory individuals. Circulation. 2007;115(11):1345-1353.

50. Harrell, Frank E. Regression Modeling Strategies, with Applications to Linear Models, Logistic and Ordinal Regression, and Survival Analysis. 2nd edition. New York, New York, USA: Springer; 2015.

51. Walker AMN, et al. Prevalence and predictors of sepsis death in patients with chronic heart failure and reduced left ventricular ejection fraction. J Am Heart Assoc. 2018;7(20):e009684

52. York MK, et al. B-type natriuretic peptide levels and mortality in patients with and without heart failure. J Am Coll Cardiol. 2018;71(19):2079-2088.

53. Guo C, Yo S, Jang W. Evaluating predictive accuracy of survival models with PROC PHREG. https://support.sas.com/ resources/papers/proceedings17/SAS0462-2017.pdf. Accessed May 31, 2019 